\title{
EFECTOS GEOGRÁFICOS Y AMBIENTALES SOBRE LA DISTRIBUCIÓN DE LAS AVES FORESTALES IBÉRICAS
}

\author{
Á. Ramírez* y J. L. Tellería*
}

\begin{abstract}
RESUMEN
El efecto península es un patrón según el cual la riqueza de especies disminuye en función de la distancia al continente. Sin embargo, este patrón es frecuentemente enmascarado por la incidencia de variables ambientales. En este trabajo estudiamos el efecto relativo de la ubicación geográfica y de un conjunto de variables ambientales (temperatura, precipitación, cobertura forestal...) sobre la distribución de la riqueza de especies de aves forestales en España peninsular. Los resultados sugieren que tanto la posición geográfica como las condiciones ambientales afectan a la distribución de la riqueza de aves. También se estudia si estos resultados varían en función de las características particulares de las áreas estudiadas. Para esta segunda aproximación se definieron dos corredores de similar longitud ubicados entre las costas atlánticas y mediterráneas y las montañas interiores. El corredor Atlántico mostró una elevada homogeneidad ambiental en toda su extensión, mientras el corredor Mediterráneo presentó un marcado gradiente desde las áreas más húmedas del norte a las condiciones más secas del sur. Los resultados muestran que en el corredor Atlántico la distancia al continente, no las condiciones ambientales, fue la principal predictora de la riqueza de especies, mientras que en el corredor Mediterráneo las variables ambientales fueron mejores predictoras de la distribución de la riqueza. Estos resultados son utilizados para discutir algunos aspectos biogeográficos y metodológicos de la distribución de aves forestales en la Península Ibérica.
\end{abstract}

Palabras clave: efecto península, aves forestales, riqueza de especies, Península Ibérica.

\begin{abstract}
Geographic and environmental effects on the distribution of Iberian forest bird richness

The peninsular effect is a biogeographical rule according to which species richness decreases as a function from the distance to the mainland base of peninsulas. However, this pattern is frequently obscured by the effect of environmental variables. In this paper we study the relative effect of the geographic location and some environmental variables on the distribution of the richness of Iberian forest birds. Results suggest that both the geographic location and some environmental variables (temperature, precipitation, forest coverage...) affect the species richness. We have also explored if these results can change according to the particular features of the study areas. To approach this, we defined two corridors of a similar length. The Atlantic corridor was quite homogeneous in the study environmental variables while the Mediterranean showed a sharp gradient from moist northern to dry south. Results showed that in the Atlantic corridor, distance to continent and not environmental variables were the main correlates to species richness while in the Mediterranean corridor environmental variables were the best correlates to richness distributions. These results are used to discuss some methodological and biogeographical aspects of the forest bird distribution across the Iberian Peninsula.
\end{abstract}

Keywords: peninsular effect, forest birds, species richness, Iberian Peninsula.

* Departamento de Zoología y Antropología Física. Facultad de Biología. Universidad Complutense. E-28040 Madrid, España. aramirez@bio.ucm.es, telleria@bio.ucm.es 


\section{Introducción}

Europa es una macro-península que ocupa el extremo occidental del Paleártico. En esta zona, la riqueza de especies de diferentes taxones disminuye hacia el oeste, un patrón que se ha interpretado como consecuencia de un efecto península a gran escala (Huntley, 1993; Blondel \& MouverChauviré, 1998; Mönkkönen, 1994; Tomiałojć, 2000; Baquero \& Tellería, 2001). Este efecto predice una disminución del número de especies hacia el extremo de las penínsulas apoyado en la teoría del equilibrio insular (Simpson, 1964; MacArthur \& Wilson, 1967). Según este planteamiento, la pérdida de especies al aumentar la distancia a las áreas fuente (continente) sería una consecuencia de las dificultades crecientes que afrontan las poblaciones para paliar por inmigración los efectos de las extinciones locales en la dinámica colonizaciones-extinciones (Brown \& Kodric-Brown, 1977; Brown \& Lomolino, 1998). A pesar de que numerosos estudios han ilustrado este patrón, el efecto península no es un fenómeno universal que pueda observarse en cualquier región y/o sobre cualquier taxón o comunidad de organismos (Busack \& Hedges, 1984; Brown, 1987; Wiggins, 1999). Es frecuente, por ejemplo, que las condiciones ambientales cambien al hacerlo la distancia al continente, enmascarando o distorsionando las predicciones del efecto península e, incluso, propiciando la aparición de patrones inversos (véase, por ejemplo, Brown \& Opler, 1990 para un efecto península inverso en mariposas tropicales en Florida). Diversos autores han señalado la existencia de otros factores como responsables últimos de estos patrones. Se ha señalado, por ejemplo, la incidencia de factores ambientales (clima, heterogeneidad ambiental, etc.; Milne \& Forman, 1986; Schwartz, 1988; Wiggins, 1999), biológicos (capacidad dispersiva, amplitud de nicho, etc.; Brown \& Opler, 1990; Wiggins, 1999) e históricos (Busack \& Hedges, 1984; Brown, 1987). Por lo tanto, parece que la distribución de la riqueza de especies a lo largo de una península será el resultado final de una combinación de efectos ambientales y geográficos.

La Península Ibérica ocupa el extremo suroccidental de la macro-península europea (Figura 1). Parece, por lo tanto, un escenario adecuado para analizar la existencia de un efecto península en la distribución de la riqueza de especies de diferentes grupos taxonómicos. Es importante señalar, además, que en este entorno geográfico se da una drástica transición climática entre los ambientes más húmedos $\mathrm{y}$ fríos del sector Atlántico y las zonas más secas y térmicas de la región Mediterránea que pueden enmascarar ese efecto geográfico. De hecho, la distribución de muchos organismos en Iberia se encuentra asociada a este cambio latitudinal en las condiciones ambientales, de modo que existe una marcada transición en la composición de especies entre los sectores Eurosiberiano y Mediterráneo (por ejemplo, Purroy, 1997; Pleguezuelos, 1997). Esto, junto con la existencia de una orografía compleja, podría difuminar el efecto de la distancia e, incluso, dar lugar a distribuciones inversas a las predichas por el efecto península en el caso de aquellos taxones de preferencias mediterráneas o de origen africano (Busack \& Hedges, 1984; Martín \& Gurrea, 1990).

En este trabajo, abordamos el estudio de la distribución de la riqueza de aves forestales que se reproducen en España peninsular. Además, estudiamos el efecto de una serie de variables geográficas y ambientales sobre dicha distribución. Este grupo animal parece particularmente apropiado para ilustrar el efecto península ya que el grueso de las poblaciones de aves forestales del Paleártico está compuesto por especies adaptadas a las condiciones ambientales del Paleártico central (Mönkkönen, 1994; Blondel \& Mouver-Chauviré, 1998). De hecho, muchas de estas aves tienden a presentar un descenso de su abundancia y una distribución crecientemente fragmentada en el suroeste del Paleártico donde, además, se observa la pérdida de ciertas especies de distribución más norteña (Tellería \& Santos, 1993; Hagemeijer \& Blair, 1997). Aunque esta tendencia podría explicarse por un efecto península, no debe descartarse el papel de determinadas restricciones ecológicas en este extremo meridional de su área de distribución paleártica (Carrascal \& Díaz, 2003, en este mismo volumen). De hecho, el aumento de la distancia a las áreas centrales de la distribución de las especies a menudo se ha relacionado con un deterioro en la calidad del hábitat y la consiguiente reducción de la abundancia de dichas especies (Brown, 1984; Lawton, 1993; Dias, 1996, véase, no obstante una revisión sobre este patrón y sus limitaciones en Sagarin \& Gaines, 2002).

Dado que el efecto relativo de los factores geográficos y ambientales puede variar según zonas (Lobo et al., 2001), hemos diseñado dos aproximaciones complementarias aprovechando ciertas particularidades topográficas de la Península Ibérica. Ésta se encuentra separada del resto del continente por los Pirineos, una gran cadena montañosa continua que presenta dos pasos en sus extremos oriental (Mediterráneo) y occidental (Atlántico; Figura 1). Estos pasos constituyen dos vías de paso de aves 

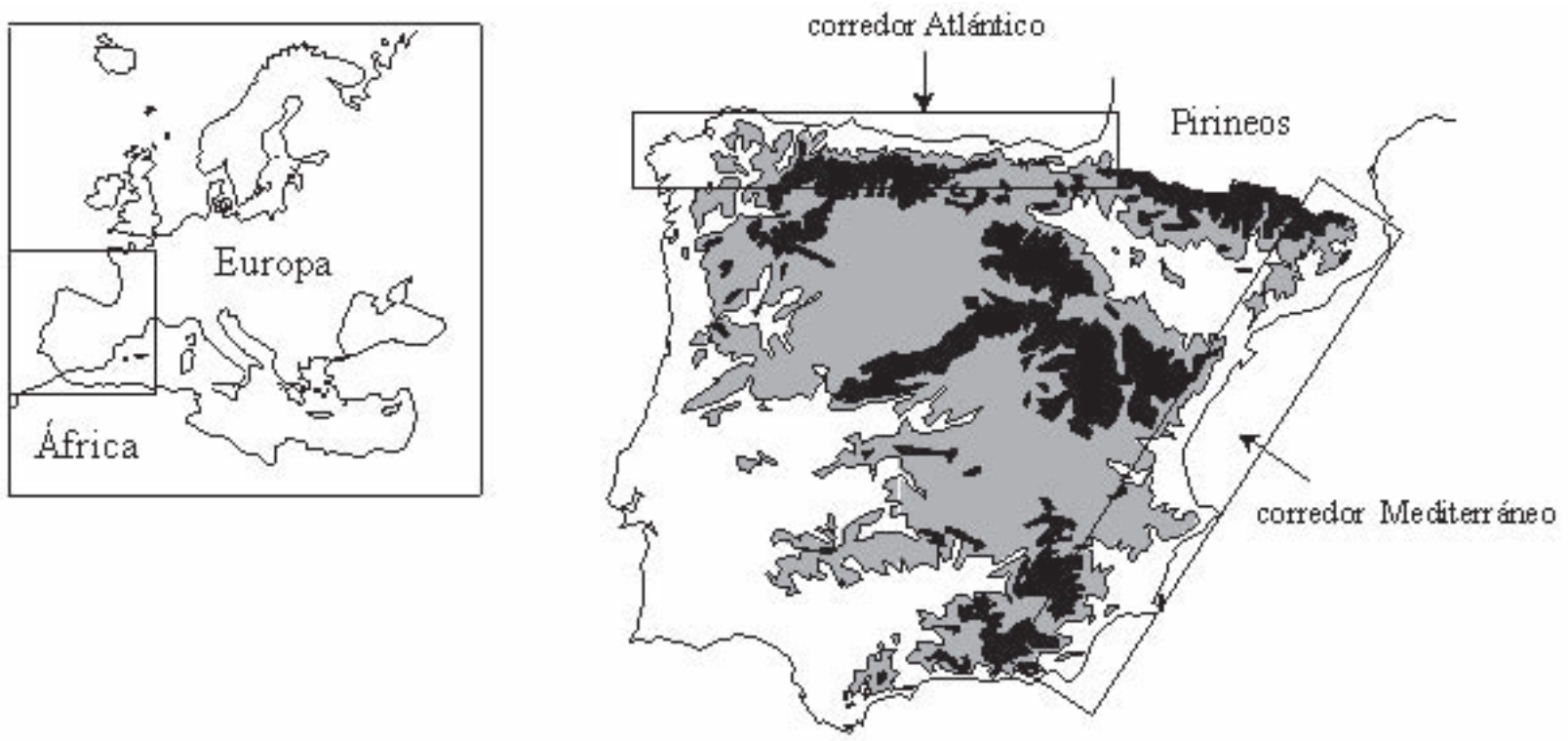

Fig. 1.- Rasgos topográficos de la Península Ibérica y situación de los corredores estudiados (en gris las áreas por encima de los 500 m.s.n.m. y en negro las ubicadas por encima de los 1000 m.s.n.m.).

Fig. 1.- Topographical features of the Iberian Peninsula and location of the studied corridors (areas over 500 m.a.s.l. in grey and those over 1.000 m.a.s.l. in black).

durante su migración (Bernis, 1963), así como sendas vías de colonización de Iberia para las especies de latitudes más norteñas, ya que conectan las tierras bajas francesas con sendos corredores definidos por las costas Atlántica y Mediterránea y los sistemas montañosos interiores (Figura 1). Históricamente dichos pasos habrían constituido igualmente vías de penetración durante los procesos de avance y retracción de los hielos pleistocénicos (Hewitt, 1999), quedando cubierta por los hielos buena parte del corredor Atlántico durante los periodos de máxima expansión glacial (Goudie, 1977). Sin embargo, estos corredores presentan características ambientales muy contrastadas, propias de las regiones Eurosiberiana (corredor Atlántico) y Mediterránea (corredor Mediterráneo) (Font, 1983). Mientras el corredor Atlántico $(600 \mathrm{~km}$ de longitud entre la frontera francesa y el Cabo de Finisterre, 74-92 km de anchura), posee unas condiciones climáticas bastante homogéneas a lo largo de toda su extensión, caracterizadas por un clima atemperado y húmedo típicamente Eurosiberiano, el corredor Mediterráneo $(650 \mathrm{~km}$ entre la frontera francesa y el Cabo de Gata, 80-130 km de anchura) muestra un gradiente climático muy acusado, con un progresivo incremento de la sequía hacia el sur y un clima típi- camente Mediterráneo (Font, 1983). Estos corredores constituyen, por lo tanto, sendos escenarios donde evaluar el papel potencial que el efecto península y las condiciones ambientales pudiesen tener sobre la distribución de las aves forestales en la Península Ibérica.

\section{Material y métodos}

\section{RiQUEZA DE ESPECIES}

Este estudio se ha centrado en el análisis de la distribución de la riqueza de especies de aves forestales en la Península Ibérica. Se trata de aquellas aves de los Ordenes Piciformes y Passeriformes que utilizan el estrato forestal como sustrato de nidificación y/o alimentación (Apéndice). Para evaluar la distribución de la riqueza en España peninsular, se contabilizó el número de especies presentes en cada cuadrícula Lambert 1:50.000 (aprox. $28,5 \times 18,5 \mathrm{~km})$ del Atlas de las Aves Reproductoras en España (Purroy, 1997). Para estudiar si las condiciones ambientales podrían dar lugar a diferentes patrones de distribución de la riqueza según las preferencias ecológicas de las especies, se realizó una clasificación adicional de 
las mismas. Para ello, se ordenaron en cuatro grupos biogeográficos en función de su distribución en el Paleártico Occidental según Hagemeijer \& Blair (1997), (véase también Tellería \& Santos, 1994 para una aproximación similar): a) especies norte$\tilde{n} a s$, aquellas que ocupan una amplia porción de Europa central y occidental y que alcanzan su límite meridional en la región Eurosiberiana de la Península Ibérica, aunque pueden penetrar en el interior de Iberia a través de las formaciones montañosas; b) especies paleárticas, que se distribuyen a lo largo y ancho del Paleártico occidental y ocupan toda la Península; c) especies suroeste-paleárticas, presentes en la Región Mediterránea y que, además de ocupar toda la Península, colonizan los sectores más cálidos del Suroeste de Europa, y d) especies mediterráneas, solo distribuidas por la Región Mediterránea y que presentan una distribución complementaria con las especies norteñas.

\section{VARIABLES AMBIENTALES Y GEOGRÁFICAS}

Se seleccionaron un conjunto de variables que definen, a gran escala, las condiciones ambientales de cada cuadrícula: Indice de Temperatura de la superficie terrestre (TEMP), Índice de Evapotranspiración (PWI), Precipitación anual (PLUVIOSIDAD) y Cobertura de bosque (BOSQUE). Los datos de las variables TEMP (canal 4 del sensor AVHRR) y PWI, fueron obtenidos del sensor AVHRR situado a bordo de satélites NOAA (NOAA-9 y NOAA-11), utilizando los valores medios de los datos de la estación de cría de las aves (Abril a Julio) desde 1985 a 1990 (excepto 1988). Los datos de precipitaciones anuales (mm/año), procedentes del Atlas Climatológico Nacional (Font, 1983), y de cobertura de bosque (\%), basados en el mapa de bosques de la Comunidad Europea (1987), se obtuvieron superponiendo los respectivos mapas temáticos al del área de estudio y estimando para cada cuadrícula un valor medio para dichas variables. Este conjunto de variables proporciona un buen indicador del clima (Guttman et al., 1997), así como del desarrollo de la cubierta forestal. Es importante señalar aquí que, de acuerdo con nuestras previsiones, el corredor Mediterráneo presentó una mayor variabilidad ambiental que el Atlántico (véase, por ejemplo, los coeficientes de variación en la Tabla 1).

Se utilizó la localización geográfica de las cuadrículas (longitud y latitud), para evaluar la posible existencia de un efecto península. Debido al fenómeno de la autocorrelación espacial en los procesos ecológicos (dos cuadrículas próximas son más parecidas entre sí que a una tercera más alejada por un efecto puramente geográfico), es necesario controlar ese contagio espacial (Legendre, 1993). Para ello, utilizamos un polinomio de tercer grado con las coordenadas geográficas (Borcard et al., 1992):

$$
\begin{gathered}
\mathrm{f}(x, y)=b_{0}+b_{1} x+b_{2} y+b_{3} x^{2}+b_{4} x y+b_{5} y^{2}+b_{6} x^{3}+ \\
+b_{7} x^{2} y+b_{8} x y^{2}+b_{9} y^{3}
\end{gathered}
$$

donde $x$ e $y$ representan longitud y latitud, respectivamente, y $b_{\mathrm{i}}$ son los coeficientes de regresión. Dichas coordenadas fueron previamente centradas en sus respectivas medias, estandarizadas y re-escaladas entre -1 y 1 respecto a la dimensión geográfica con mayor variación (Longitud, LON), recalculando el rango de variación de la otra componente geográfica (Latitud, LAT) respecto a la Longitud estandarizada (Carrascal et al., 2002).

Aquellas cuadrículas mal muestreadas en la realización del atlas (Purroy, 1997) o sin datos meteorológicos o de satélite fueron excluidas. Para evitar posibles sesgos en la riqueza de especies asociados a diferencias en el tamaño del área muestreada, se excluyeron también las cuadrículas con más del $70 \%$ de su superficie ocupada por mar o situada fuera de España. De acuerdo con estos criterios, se consideraron un total de 857 cuadrículas para el conjunto de España peninsular, 103 cuadrículas para el corredor Atlántico y 103 cuadrículas para el corredor Mediterráneo.

\section{ANÁLISIS}

Para sintetizar y controlar los patrones de covariación entre las variables ambientales se realizó un Análisis de Componentes Principales (ACP) con las cuatro variables ambientales cuantificadas. La distribución de la riqueza de especies forestales se analizó mediante regresión múltiple utilizando modelos generales lineales, con las componentes obtenidas en el ACP y los nueve términos del polinomio de la latitud-longitud como variables independientes. Por último, con el objeto de evaluar si los patrones observados eran debidos a un efecto península (geográfico) o a las condiciones ambientales, se realizaron regresiones múltiples por pasos hacia delante (Forward) y hacia detrás (Backward) en los dos corredores definidos. En este último análisis, sólo se utilizaron las componentes ambientales obtenidas en el ACP y la distancia al punto de entrada al corredor (medida como longitud y latitud en los corredores Atlántico y Mediterráneo, respectivamente), ya que esta era la única componente geográfica con un rango de variación significativo. Todos los análisis se realizaron tanto para el conjunto de especies, como para cada uno de los cuatro grupos biogeográficos definidos. 
Tabla 1.- Caracteristicas de las variables ambientales y geográficas de los corredores Atlántico y Mediterráneo. CV: coeficiente de variación.

Table 1.- Environmental and geographical features of Atlantic and Mediterranean corridors. CV: variation coefficient.

\begin{tabular}{llrrrrr}
\hline & & PWI & TEMP & PLUVIOS & BOSQUE & DIST \\
\hline \multirow{2}{*}{ Media } & Atlántico & 132,59 & 159,64 & 1411,65 & 24,95 & 293,65 \\
& Mediterráneo & 149,42 & 171,25 & 553,40 & 30,97 & 293,37 \\
CV & Atlántico & 1,88 & 1,33 & 30,75 & 49,52 & 58,91 \\
& Mediterráneo & 4,61 & 5,21 & 47,65 & 71,56 & 62,50 \\
\multirow{2}{*}{ Mínimama } & Atlántico & 138,38 & 163,50 & 2200 & 70 & 599,98 \\
& Mediterráneo & 160,20 & 187,00 & 1250 & 90 & 645,93 \\
& Atlántico & 126,25 & 155,13 & 550 & 10 & 18,53 \\
& Mediterráneo & 126,33 & 154,83 & 200 & 0 & 0 \\
\hline
\end{tabular}

\section{Resultados}

\section{PATRONES GEOGRÁFICOS}

La máxima riqueza de aves forestales se encuentra en las áreas más próximas a Europa continental, observándose una pérdida gradual de especies hacia las áreas más meridionales, donde sólo las áreas montañosas parecen mantener valores importantes (Figura 2a). Sin embargo, existen diferencias entre grupos biogeográficos. Mientras el número de especies norteñas, paleárticas y suroeste-paleárticas siguió este mismo patrón (Fig. 2b-d), las especies mediterráneas mostraron un patrón opuesto al aumentar su riqueza hacia el Sur (Fig. 2e).

\section{EFECTOS AMBIENTALES Y GEOGRÁFICOS}

El Análisis de Componentes Principales dio lugar a dos componentes que parecen definir sendos gradientes ambientales (Tabla 2). La primera componente (PC1) puede interpretarse como un gradiente climático que diferencia aquellas áreas más cálidas y secas (valores positivos de TEMP y PWI), de las zonas menos térmicas y con mayores precipitaciones (menores valores de Pluviosidad). Esta componente parece describir bien el gradiente climático resultante de la transición EurosiberianoMediterránea característico de la Península Ibérica. La segunda componente (PC2) se asoció principal y negativamente con la variable BOSQUE, separando en un extremo las cuadrículas con menor superficie forestal, de aquellas con mayor cobertura de bosque.

A escala peninsular la riqueza de especies se asoció tanto a los factores ambientales como a los geográficos. El número de especies mostró una asociación negativa con el PC1, indicando una disminución del número de especies con el incremento de la temperatura y la xericidad. De igual manera se observó una asociación negativa con la segunda componente (PC2), lo que indicaría que la riqueza aumentó con la cobertura de bosque. Por grupos de especies, como era de esperar, sólo las especies mediterráneas mostraron un patrón opuesto, asociándose positivamente con la componente climática (PC1). Igualmente, la ubicación geográfica juega un papel relevante en la distribución de la riqueza de aves forestales. Aunque con ciertas variaciones según los grupos de especies, en términos generales se obtuvieron mayores valores de riqueza hacia el norte de España (asociación positiva con la latitud), con ligeros aumentos tanto en los extremos sur, suroeste, este y noreste de la península (Tabla 3), y valores más bajos en las cuadrículas centrales. La habilidad del conjunto de variables seleccionadas para ilustrar los patrones de distribución de la riqueza de especies disminuyó de las aves norte-

Tabla 2.- Análisis de Componentes Principales realizado con las variables ambientales consideradas en este estudio. $(* \mathrm{p}<0,05 ; \mathrm{n}=857)$.

Table 2.- Principal Components Analysis performed with the environmental variables considered in this study. $\left({ }^{*} \mathrm{p}<0.05 ; \mathrm{n}=857\right)$.

\begin{tabular}{lcc}
\hline Variable & PC1 & PC2 \\
\hline PWI & $0,882^{*}$ & $-0,066$ \\
TEMP & $0,871^{*}$ & $-0,150^{*}$ \\
PLUVIOSIDAD & $-0,847^{*}$ & $-0,004$ \\
BOSQUE & $-0,190^{*}$ & $-0,979^{*}$ \\
\hline Autovalor & 2,291 & 0,986 \\
Varianza explicada (\%) & 57,3 & 24,7 \\
Varianza acumulada (\%) & 57,3 & 82,0 \\
\hline
\end{tabular}




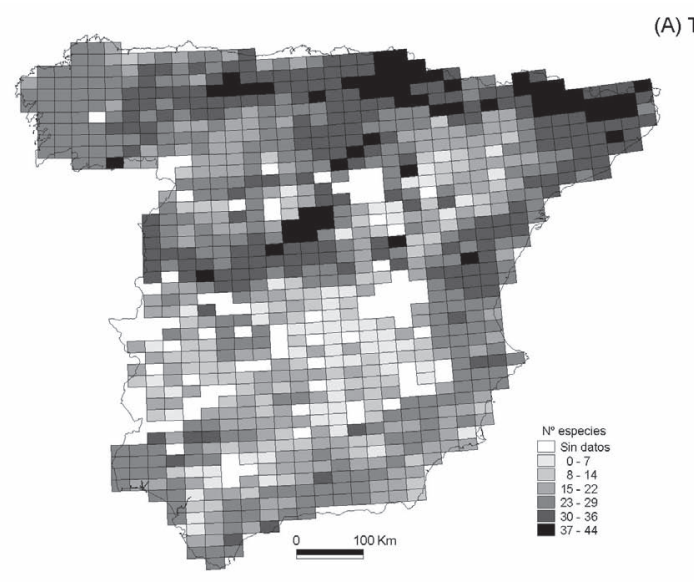

(A) Total

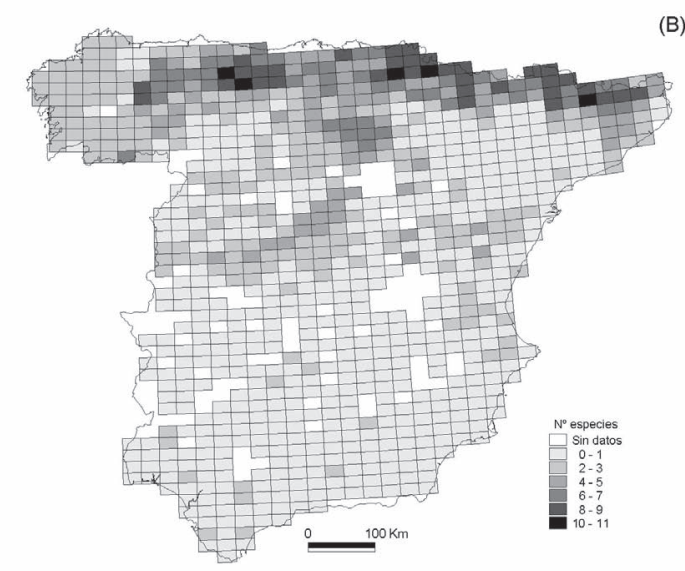

(B) Norteñas
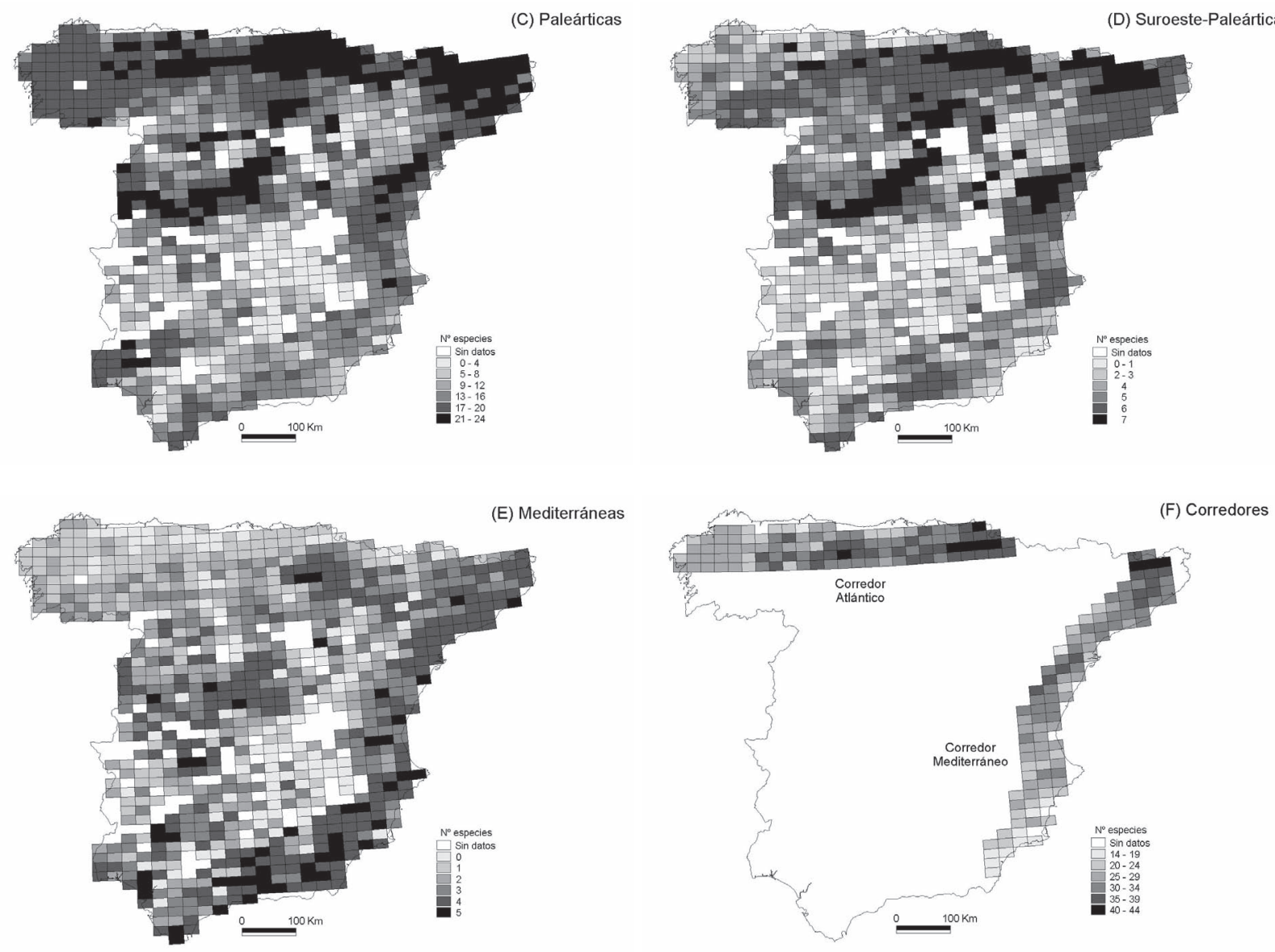

Fig. 2.- Distribución de la riqueza total de especies de aves forestales en España y de los cuatro grupos biogeográficos definidos: A) Total, B) especies norteñas, C) especies paleárticas, D) especies suroeste-paleárticas, E) especies mediterráneas. Se indican también las cuadrículas contenidas en los corredores Atlántico y Mediterráneo (F).

Fig. 2.- Distribution of the total richness of forest birds in Spain as well as the richness of the four biogeographical groups: A) Total, B) northern species, C) palaearctic species, D) south-western palaearctic species, and E) mediterranean species. The distribution of the squares contained in the Atlantic and Mediterranen corridors is also shown (F). 
Tabla 3.- Análisis de Regresión Múltiple entre el número total de especies y el de cada uno de los grupos biogeográficos definidos y las variables ambientales y geográficas. Se indica el coeficiente de regresión (B) y el valor del estadístico (F) para cada una de las variables. También se incluye el porcentaje de varianza explicada $\left(\mathrm{R}^{2}\right)$ por cada uno de los modelos $\left(* * \mathrm{p}<0,001 ;{ }^{*} \mathrm{p}<\right.$ $0,05 ; \mathrm{n}=857)$.

Table 3.- Multiple Regression analyses between total richness and richness of the four biogeographic groups and the environmental and geographic variables. Regression coefficients (B) and F-value (F) are shown. The explained variance $\left(\mathrm{R}^{2}\right)$ is also shown $(* * \mathrm{p}<0.001 ; * \mathrm{p}<0.05 ; \mathrm{n}=857)$.

\begin{tabular}{|c|c|c|c|c|c|c|c|c|c|c|}
\hline & \multicolumn{2}{|c|}{ Mediterráneas } & \multicolumn{2}{|c|}{ SO-Paleárticas } & \multicolumn{2}{|c|}{ Paleárticas } & \multicolumn{2}{|c|}{ Norteñas } & \multicolumn{2}{|c|}{ Total } \\
\hline & B & $\mathbf{F}$ & B & $\mathbf{F}$ & B & $\mathbf{F}$ & B & $\mathbf{F}$ & B & $\mathbf{F}$ \\
\hline PC1 & $0,21 *$ & 5,4 & $-0,96 * *$ & 81,0 & $-3,86^{* *}$ & 159,8 & $-1,67 * *$ & 325,9 & $-6,27 * *$ & 160,23 \\
\hline PC2 & $-0,18 *$ & 11,6 & $-0,16^{*}$ & 6,3 & $-0,69 * *$ & 14,6 & 0,09 & 2,9 & $-0,94 *$ & 10,31 \\
\hline LON & $0,52 *$ & 5,4 & 0,48 & 3,4 & $1,70^{*}$ & 5,2 & $-0,43$ & 3,6 & 2,27 & 3,54 \\
\hline LAT & $1,55 * *$ & 15,8 & $4,09 * *$ & 81,8 & $6,59 * *$ & 26,3 & $-0,56$ & 2,1 & $11,67 * *$ & 31,17 \\
\hline $\mathrm{LON}^{2}$ & $2,82 * *$ & 40,9 & $2,99 * *$ & 34,1 & $15,26^{* *}$ & 109,9 & $1,06^{*}$ & 5,8 & $22,13 * *$ & 87,50 \\
\hline LAT $^{2}$ & $4,58 * *$ & 33,4 & $3,14^{*}$ & 11,7 & $11,03 * *$ & 17,7 & $3,70 * *$ & 21,7 & $22,46 * *$ & 27,83 \\
\hline LONxLAT & $-4,49 * *$ & 44,2 & $-5,16^{* *}$ & 43,4 & $-16,38^{* *}$ & 54,0 & 0,20 & 0,1 & $-25,83 * *$ & 50,80 \\
\hline LON $^{2} x$ LAT & $-4,43 * *$ & 16,0 & $-8,76^{* *}$ & 46,6 & $-36,78^{* *}$ & 101,1 & $-5,71 * *$ & 26,6 & $-55,68^{* *}$ & 87,81 \\
\hline LONxLAT $^{2}$ & $7,38 * *$ & 23,3 & $9,49 * *$ & 28,7 & $20,91 * *$ & 17,2 & $10,94 * *$ & 51,3 & $48,72 * *$ & 35,31 \\
\hline $\mathrm{LON}^{3}$ & 0,84 & 3,8 & 0,78 & 2,5 & $3,54^{*}$ & 6,3 & 0,36 & 0,7 & $5,51^{*}$ & 5,79 \\
\hline LAT $^{3}$ & $-15,17 * *$ & 83,4 & $-20,87^{* *}$ & 117,5 & $-27,76^{* *}$ & 25,6 & $9,67 * *$ & 33,9 & $-54,13^{* *}$ & 36,92 \\
\hline $\mathrm{R}^{2}(\%)$ & \multicolumn{2}{|c|}{26,8} & \multicolumn{2}{|c|}{36,0} & \multicolumn{2}{|c|}{49,4} & \multicolumn{2}{|c|}{68,9} & \multicolumn{2}{|c|}{49,4} \\
\hline
\end{tabular}

ñas y paleárticas a las suroeste-paleárticas y mediterráneas que fueron las peor modelizadas, como puede apreciarse en los valores de varianza explicada (Tabla 3).

\section{CORREDORES}

Los dos corredores mostraron un descenso en la riqueza de especies hacia las zonas más alejadas del centro del continente (Fig. 3). Sin embargo, el porcentaje de varianza explicada por los gradientes geográficos y ambientales fue diferente en los dos corredores. En el corredor Atlántico, la componente geográfica (longitud) tuvo mayor importancia cuantitativa en la varianza explicada $(46,2 \%)$, mientras que las variables ambientales sólo explicaron un 3,1\% (PC2) (Tabla 4), por lo que puede concluirse que la componente geográfica fue la principal responsable del patrón observado. Todos los grupos de especies mostraron igualmente una asociación positiva con la longitud, siendo la componente geográfica la que retuvo la mayor parte de la varianza explicada, y quedando en un segundo plano las variables climáticas. Sólo en las especies mediterráneas, la componente climática $(\mathrm{PC} 1)$ fue la que retuvo mayor porcentaje de la varianza (Tabla 4), mostrando nuevamente una asociación positiva con dicha componente. Esto parece reflejar diferencias en la capacidad de colonización de cada uno de los grupos asociadas a sus preferencias de hábitat. En el corredor Mediterráneo, la riqueza total de especies disminuyó hacia el extremo del mismo (Figura $3 b$ ). Sin embargo en este corredor, la única variable que apareció como significativa fue la componente climática (PC1), que explicó un $74 \%$ de la varianza para el conjunto de especies. Por grupos de especies el patrón general es el mismo, con una preponderancia de la componente climática sobre la geográfica (2-3\% de la varianza explicada) (Tabla 4). Esto parece apoyar la idea de que el número de especies de aves forestales disminuyó más por el efecto de la temperatura y la sequía que por la lejanía al continente. De nuevo las especies mediterráneas mostraron un patrón opuesto, asociándose positivamente con la componente climática, y volviendo a ser las peor modelizadas (6,2\% de la varianza explicada).

\section{Discusión}

Pese a la diversidad de factores determinantes de la distribución de la riqueza de especies en una región, siempre es útil conocer quienes son los responsables de la mayor parte de la variación observada (Currie, 1991; Lobo et al., 2001). Esto permite avanzar en el conocimiento de las restricciones ambientales a las que están sometidas las especies así como interpretar el potencial efecto de la histo- 

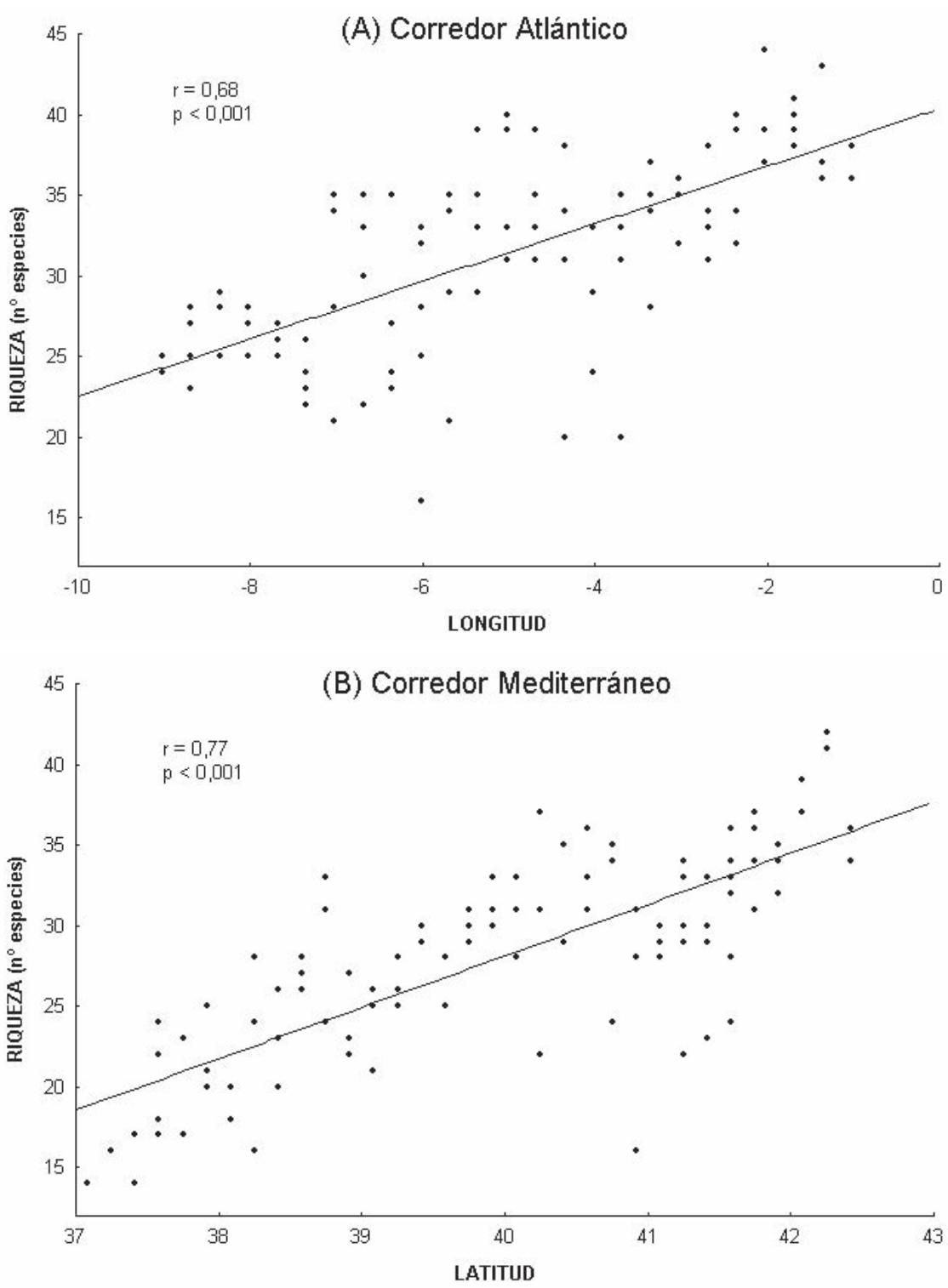

Fig. 3.- Correlación entre la riqueza total de aves forestales y la distancia a los Pirineos en los dos corredores analizados (Atlántico y Mediterráneo).

Fig. 3. - Correlation between the total richness of forest birds and the distance to Pyrenees in Atlantic (A) and Mediterranean (B) corridors.

ria sobre su distribución actual. Hay que tener en cuenta, sin embargo, que estas aproximaciones están condicionadas por una serie de limitaciones operativas que restringen la generalización de las conclusiones obtenidas. Estas tienen que ver, en primer lugar, con la escala de estudio ya que los factores que afectan a la riqueza de especies, y su interpretación biogeográfica, pueden cambiar según se aborden a nivel regional o continental (Brown \& Lomolino, 1998). En segundo lugar, es importante considerar el escenario en el que se han obtenido los resultados, ya que los rangos de variación de los factores que afectan a las especies pueden cambiar entre regiones con la consiguiente dis- 
Tabla 4.- Análisis de Regresión Múltiple por pasos hacia delante entre la riqueza (total y por grupos) y las variables ambientales y geográficas en los corredores estudiados (Atlántico, $n=103)$ y Mediterráneo $(n=103)$. Sólo se han incluido las variables que entraron como significativas $(\mathrm{p}<0,05)$ en los modelos. Se indican los valores de los coeficientes de regresión y la varianza explicada por cada una de las variables (entre paréntesis).

Table 4.- Forward Stepwise Regression analyses between richness and environmental and geographic variables at the two corridors (Atlantic, $n=103$; and Mediterranean, $n=103$ ). Only significant variables $(\mathrm{p}<0.05)$ included in the models are shown. Regression coefficients and the explained variance (in brackets) are also shown.

\begin{tabular}{|c|c|c|c|c|c|}
\hline & Intercepto & LONGITUD & PC1 & PC2 & $R^{2}(\%)$ \\
\hline Mediterráneas & 3,02 & & $1,033(27,6)$ & $-0,526(14,0)$ & 41,6 \\
\hline SO-Paleárticas & 7,88 & $0,245(24,9)$ & $0,920(6,0)$ & $-0,795(5,9)$ & 36,8 \\
\hline Paleárticas & 23,26 & $0,635(34,8)$ & & & 34,8 \\
\hline Norteñas & 5,61 & $0,773(37,6)$ & $-2,220(17,3)$ & & 54,9 \\
\hline Total & 40,06 & $1,595(46,2)$ & & $-1,764(3,1)$ & 49,3 \\
\hline
\end{tabular}

Corredor Mediterráneo

\begin{tabular}{|c|c|c|c|c|c|}
\hline & Intercepto & LATITUD & PC1 & PC2 & $R^{2}(\%)$ \\
\hline Mediterráneas & 3,695 & & $0,229(6,2)$ & & 6,2 \\
\hline SO-Paleárticas & $-4,090$ & $0,244(2,3)$ & $-0,673(44,1)$ & & 46,4 \\
\hline Paleárticas & $-12,250$ & $0,763(2,5)$ & $-2,518(64,0)$ & & 66,5 \\
\hline Norteñas & 18,950 & $-0,410(2,9)$ & $-2,507(66,2)$ & & 69,1 \\
\hline Total & 30,550 & & $-6,372(74,0)$ & & 74,0 \\
\hline
\end{tabular}

paridad en la respuesta de la riqueza a una variable geográfica o ambiental determinada (Kerr \& Packer, 1997). Y, finalmente, han de tenerse en cuenta las características biológicas de las especies analizadas ya que los resultados basados en el estudio de la distribución de un gremio o comunidad no tienen por qué ser extrapolables a otros grupos de especies (Kerr, 1997; Prendergast et al., 1993; Waide et al., 1999). Por eso, puede ser útil discutir los resultados desde esta triple perspectiva (escala geográfica, características regionales y composición taxonómica).

\section{ESCALA GEOGRÁFICA}

La distribución de las aves forestales ibéricas es el resultado de las fluctuaciones ambientales que tuvieron lugar en el Paleártico Occidental durante el Cuaternario. A lo largo del Pleistoceno tardío, y a medida que el calentamiento global fue progresando tras la retirada de los hielos y la sequía estival se fue extendiendo desde las áreas más meridionales hacia el norte, el óptimo ecológico de los bosques caducifolios y de coníferas se fue desplazando hacia el centro de Europa (Huntley, 1993; Hewitt, 1999), con la consiguiente retirada de muchas aves forestales (Blondel \& MouverChauviré, 1998). En el área mediterránea, estos cambios fueron acompañados de una severa y continua presión humana (Lobo et al., 2001) que favoreció a las especies vegetales heliófilas, esclerófilas y pirófitas, con la consiguiente expansión de las aves asociadas a estos medios más secos y arbustivos (Costa et al., 1990; Blondel \& Vigne, 1993). La Península Ibérica, donde la transición de las regiones Eurosiberiana y Mediterránea replica a menor escala estos cambios ambientales continentales, es un escenario amplio y contrastado donde, además, encuentran el limite de su área de distribución muchas aves forestales norteñas (Tellería \& Santos, 1993). Esto no se da en otros sectores centroeuropeos donde, tal y como queda reflejado en la estructura y composición de la vegetación (Ozenda et al., 1979), el ambiente es mucho más uniforme y la distribución de las aves no ilustra patrones tan claros como los aquí descritos (Blackburn et al., 1999).

Es también importante resaltar desde esta perspectiva el posible origen de los patrones de distribución peninsular observados. De acuerdo con lo arriba expuesto, la distribución actual de las aves forestales ibéricas parece deberse más a los resultados de un proceso de retracción que a un clásico evento de colonización peninsular desde el continente próximo. Esto no altera, sin embargo, la lógica del efecto península (asimetría en la dinámica de 
extinciones y colonizaciones en poblaciones periféricas y crecientemente alejadas del grueso poblacional) ni la posibilidad de que la distribución actual de ciertas especies sea el resultado de eventos de colonización recientes no conocidos. Después de todo, ciertas especies de árboles típicamente centroeuropeos (el haya Fagus sylvatica) parecen haber irrumpido en el escenario ibérico al retirarse las glaciaciones (Costa et al., 1990).

\section{CARACTERÍSTICAS REGIONALES}

Los resultados obtenidos en este trabajo sugieren que la riqueza de especies de aves forestales de España peninsular está condicionada tanto por el ambiente como por la distancia al continente, variando la importancia de estas según regiones. Esta última parece tener una importancia menor que la calidad ambiental (precipitaciones, desarrollo del bosque...) pues explica mucha menos varianza de la distribución de la riqueza (Tabla 2). Ese papel secundario de la distancia se ha observado, por ejemplo, en la distribución de la riqueza de plantas leñosas en un conjunto de penínsulas de Norteamérica (Milne \& Forman, 1986), y de plantas vasculares en la Península Ibérica (Lobo et al., 2001). En el contexto ibérico, esto puede relacionarse con los mencionados cambios ambientales drásticos asociados a la transición bioclimática entre las regiones Eurosiberiana y Mediterránea que provocan el paso desde los húmedos bosques de los Pirineos o de la Cordillera Cantábrica a las secas formaciones esclerófilas del sureste árido. Parece razonable aceptar, por lo tanto, que de no darse estas contrastadas condiciones ambientales, el efecto de la distancia hubiera sido más acentuado. Afortunadamente, los resultados obtenidos en los corredores parecen corroborar esta interpretación. Mientras que los factores que determinan la distribución de las aves forestales en el corredor Mediterráneo son principalmente ambientales (Tabla 4), la distribución de la riqueza en el corredor Atlántico, mucho más homogéneo ambientalmente (Tabla 1), viene determinada básicamente por la distancia a la zona de contacto con el continente (Tabla 4).

\section{COMPOSICIÓN TAXONÓMICA}

El trabajo ilustra claramente que diferentes grupos de especies responden de forma diferente a la variación ambiental de la Península Ibérica. La mayoría de las aves forestales se asocian mejor con los sectores más húmedos y boscosos del norte o de las montañas ibéricas que con las zonas más secas de la mitad meridional (Carrascal \& Díaz, 2003, en este mismo volumen). Esto es lógico si consideramos las peculiaridades ecológicas del grupo estudiado y el hecho de que en estas últimas zonas el arbolado tiende a desarrollarse mal y a defenderse peor del secular proceso de deterioro de la cubierta forestal ibérica (Costa et al., 1990). En los bosques aclarados y de facies arbustiva típicos de amplios sectores del mediterráneo ibérico, muchas especies de aves forestales comunes crían mal (por ejemplo, Erithacus rubecula, Sylvia atricapilla, Phylloscopus collybita, Sylvia borin, Turdus philomelos), y suelen presentar una condición física deficiente con respecto a sus poblaciones de los bosques más norteños (Carbonell \& Tellería, 1998, 1999; Pérez-Tris et al., 2000) y, a menudo, terminan por desaparecer de grandes regiones (Tellería \& Santos, 1993; Purroy, 1997). Por el contrario, aquellas especies adaptadas a ambientes más xéricos y deforestados (Sylvia cantillans, S. hortensis, S. undata, Phylloscopus bonelli, Serinus seri$n u s)$ tienden a desarrollarse mejor. Esta interpretación parece quedar corroborada por el desigual sentido de la asociación de la riqueza de aves forestales paleártico-norteñas frente a las mediterráneas. Mientras que la riqueza de estas últimas, más adaptadas a los ambientes típicamente mediterráneos, aumenta en los sectores más térmicos y menos húmedos, las primeras parecen encontrar fuertes limitaciones ambientales en ese tipo de medios y se desarrollan mejor en los bosques húmedos y más desarrollados de ambientes eurosiberianos. Estas diferencias en las preferencias ecológicas explicarían que las especies mediterráneas se desmarquen de los patrones obtenidos para el resto de grupos. Además, la posición periférica de la Península Ibérica en las áreas de distribución de las especies estudiadas, podría estar relacionada con los patrones de distribución de riqueza observados (Brown, 1984; Tellería \& Santos, 1993). Esto, y no el efecto península, unido al deterioro de las condiciones ambientales óptimas para las especies mediterráneas, explicaría la pérdida de riqueza de este grupo en el corredor atlántico. Por lo tanto, y pese a su carácter mayoritariamente norteño, las aves forestales ibéricas no son un grupo homogéneo en su respuesta a las condiciones ambientales que determinan la distribución de su riqueza de especies.

Un comportamiento igualmente dispar puede encontrarse en las mariposas ibéricas, donde la pérdida de riqueza hacia el sur para el conjunto de especies (principalmente de origen europeo) se incumple en los grupos de origen africano (Antocharinae) que tienden a diversificarse en las áreas más meridionales (Martín \& Gurrea, 1990). Estas diferencias en los patrones de distribución también se pueden 
observar en otros grupos biológicos, como los lagartos y serpientes (Busack \& Hedges, 1984), que aumentan su riqueza de especies en las zonas más térmicas del sur peninsular. Por lo tanto, aunque existe un claro efecto de la distancia en los patrones de distribución de la riqueza de las aves forestales a lo largo de la Península Ibérica, su efecto parece estar claramente condicionado por la identidad taxonómica y preferencias ecológicas de estas especies.

\section{AGRADECIMIENTOS}

Los comentarios de L. M. Carrascal contribuyeron a mejorar el manuscrito original. J. T. García y J. Pérez-Tris, aportarton valiosas sugerencias durante el análisis de datos.

\section{Referencias}

Baquero, R. A. \& Tellería, J. L., 2001. Species richness, rarity and endemicity of European mammals: a biogeographical approach. Biodiversity and Conservation, 10: 29-44.

BERNIS, F. 1963., Sobre migración de nuestros passeriformes transaharianos. Ardeola, 8: 41-119.

Blackburn, T. M., Gaston, K. J., Quinn, R. M. \& GREGORY, R. D., 1999. Do local abundances of British birds change with proximity to range edge? Journal of Biogeography, 26: 493-505.

Blondel, J. \& Vigne, J. D., 1993. Space, time, and man as determinants of diversity of birds and mammals in the Mediterranean region. In: R.S. Ricklefs \& D. Schluter (eds.). Species Diversity in Ecological Communities. University of Chicago Press. Chicago: 135-146.

Blondel, J. \& Mouver-Chauviré, C., 1998. Evolution and history of the Western Palearctic avifauna. Trends in Ecology and Evolution, 13: 488-492.

Bocard, D., Legendre, P. \& Drapeau, P., 1992. Partialling out the spatial component of ecological variation. Ecology, 73: 1045-1055.

Brown, J. H., 1984. On the relationship between abundance and distribution of species. American Naturalist, 124: 253-279.

Brown, J. H. \& Kodric-Brown, A., 1977. Turnover rates in insular biogeography: effect of immigration on extinction. Ecology, 58: 445-449.

Brown, J. H. \& Lomolino, M. V., 1998. Biogeography. $2^{\mathrm{a}} \mathrm{ed}$. Sinauer. Sunderland. $691 \mathrm{pp}$.

Brown, J. W., 1987. The peninsular effect in Baja California: an entomological assessment. Journal of Biogeography, 14: 359-365.

Brown, J. W. \& Opler, P. A., 1990. Patterns of butterfly species density in peninsular Florida. Journal of Biogeography, 17: 615-622.
BusACK, S. D. \& Hedges, S. B., 1984. Is the peninsular effect a red herring? American Naturalist, 123: 266275.

Carbonell, R. \& Tellería, J. L., 1998. Selección de hábitat de cinco poblaciones ibéricas de Curruca Capirotada (Sylvia atricapilla). Ardeola, 45: 1-10.

Carbonell, R. \& Tellería, J. L., 1999. Feather traits and ptilochronology as indicators of stress in Iberian Blackcaps (Sylvia atricapilla). Bird Study, 46: 243-248.

CArrascal, L. M. \& DíAz, L., 2003. Asociación entre distribucióm continental y regional. Análisis con la avifauna forestal $\mathrm{y}$ de medios arbolados de la Península Ibérica. Graellsia, 59(2-3): 179-207.

Carrascal, L. M., Palomino, D. \& Lobo, J. M., 2002. Patrones de preferencias de hábitat y de distribución y abundancia invernal de aves en el centro de España. Análisis y predicción del efecto de factores ecológicos. Animal Biodiversity \& Conservation, 25: 7-40.

Costa, M., García, M., Morla, C. \& SÁinz, H., 1990. La evolución de los bosques de la Península Ibérica: una interpretación basada en datos paleobiogeográficos. Ecología, 1: 31-58.

Currie, D. J., 1991. Energy and large-scale patterns of animal- and plant-species richness. American Naturalist, 137: 27-49.

DiAs, P. C., 1996. Sources and sinks in population biology. Trends in Ecology and Evolution, 11: 326-330.

Font, I., 1983. Climatología de España y Portugal. Instituto Nacional de Meteorología. Madrid. 296 pp.

Goudie, A. S., 1977. Environmental change. Oxford Univesity Press. Oxford. 244 pp.

Guttman, G., Tarpley, D., Ignatov, A. \& Olson, S., 1997. Global AVHRR-Derived Land Climatology. National Oceanic and Atmospheric Administration. Boulder. CD-Rom.

HagemeiJer, W. \& Blair, M. J., 1997. The EBCC Atlas of European Breeding Birds. T \& AD Poyser. London. 903 pp.

Hewitt, G. M., 1999. Post-glacial re-colonization of European biota. Biological Journal of the Linnean Society, 68: 87-112.

Huntley, B., 1993. Species-richness in north-temperate zone forests. Journal of Biogeography, 20:163180.

KerR, J. T., 1997. Species richness, endemism, and the choice of areas for conservation. Conservation Biology, 11: 1094-1100.

KerR, J. T. \& PACKER, L., 1997. Habitat heterogeneity as a determinant of mammal species richness patterns in high-energy regions. Nature, 385: 252-254.

LAWTON, J. H., 1993. Range, population abundance and conservation. Trends in Ecology and Evolution, 8: 409-413. 
LEGENDRE, P., 1993. Spatial autocorrelation: trouble or new paradigm? Ecology, 74: 1659-1673.

Lobo, J. M., CAstro, I. \& Moreno, J. C., 2001. Spatial and environmental determinants of vascular plant species richness distribution in the Iberian Peninsula and Balearic Islands. Biological Journal of the Linnean Society, 73: 233-253.

MacArthur, R. H. \& Wilson, E. O., 1967. The theory of island biogeography. Princeton University Press. Princeton. 203 pp.

Martín, J. \& Gurrea, P., 1990. The peninsular effect in Iberian butterflies (Lepidoptera: Papilionoidea and Hesperioidea). Journal of Biogeography, 17: 85-96.

Milne, B. T. \& Forman, T. T., 1986. Peninsulas in Maine: Woody plant diversity, distance, and environmental patterns. Ecology, 67: 967-974.

MÖNKKÖNEN, M., 1994. Diversity patterns in Palaearctic and Nearctic forest bird assemblages. Journal of Biogeography, 21: 183-195.

Ozenda, P., Noirfalise, A. \& Trautmann, W., 1979. Carte de la Végétation des états membres du Conseil de L'Europe. Conseil de l'Europe. Strasbourg. 53 pp.

Pérez-Tris, J., Carbonell, R. \& Tellería, J. L., 2000. Abundance distribution, morphological variation, and juvenile condition of robins Erithacus rubecula in their Mediterranean range boundary. Journal of Biogeography, 27: 879-888.

Pleguezuelos, J. M. (ed.), 1997. Distribución y Biogeografía de los Anfibios y Reptiles en España y Portugal. Universidad de Granada. Granada. 542 pp.

Prendergast, J. R., Quinn, R. M., Lawton, J. H., Eversham, B. C. \& GibBons, D. W., 1993. Rare species, the coincidence of diversity hotspots and conservation strategies. Nature, 365: 335-337.
Purroy, F. J. (ed.), 1997. Atlas de las Aves de España (1975-1995). Lynx. Barcelona. 583 pp.

SAgARIN, R. D. \& GAINES, S. D., 2002. The "abundance centre" distribution: to what extent is it a biogeographical rule? Ecology Letters, 5: 137-147.

SChWARTZ, M. W., 1988. Species diversity patterns in woody flora on three North American peninsulas. Journal of Biogeography, 15: 759-774.

Simpson, G. G., 1964. Species density of North American recent mammals. Systematic Zoology, 13: 57-73.

Tellería, J. L. \& Santos, T., 1993. Distributional pattern of insectivorous passerines in the Iberian forests: does abundance decrease near the border? Journal of Biogeography, 20: 235-240.

TelleríA, J. L. \& SANTos, T., 1994. Factors involved in the distribution of forest birds in the Iberian Peninsula. Bird Study, 41: 161-169.

TomiaŁoJ'́, L., 2000. An East-West gradient in the breeding distribution and species richness of the European woodland avifauna. Acta Ornithologica, 35(1): 3-17.

Waide, R. B., Willig, M. R., Steiner, C. F., Mittelbach, G., Gough, L., Dodson, S. I., Juday, G. P. \& PARmenter, R., 1999. The relationship between productivity and species richness. Annual Review of Ecology and Systematic, 30: 257-300.

Wiggins, D. A., 1999. The peninsula effect on species diversity: a reassessment of the avifauna of Baja California. Ecography, 22: 542-547. 
Apéndice.- Listado de especies incluidas en el presente estudio. Se indican los grupos biogeográficos definidos para cada una de las especies: M (Mediterráneas), SW (Suroeste-Paleárticas), P (Paleárticas) y N (Norteñas). También se indica el número de cuadrículas ocupadas en el total del área de estudio (Cuadrículas).

Appendix.- L List of species included in this study. Biogeographic group: M (Mediterranean), SW (South-western palaearctic), P (Palaearctic) and $\mathrm{N}$ (Northern) species (see text). The number of occupied squares (Cuadrículas) by each specie is also shown.

\begin{tabular}{|c|c|c|c|c|c|}
\hline ESPECIES & Grupo & Cuadrículas & ESPECIES & Grupo & Cuadrículas \\
\hline Jynx torquilla & $\mathrm{P}$ & 401 & Phylloscopus collybita & $\mathrm{P}$ & 482 \\
\hline Picus viridis & $\mathrm{P}$ & 707 & Phylloscopus trochilus & $\mathrm{N}$ & 32 \\
\hline Dryocopus martius & $\mathrm{N}$ & 49 & Regulus regulus & $\mathrm{N}$ & 161 \\
\hline Dendrocopos major & $\mathrm{P}$ & 530 & Regulus ignicapillus & SW & 461 \\
\hline Dendrocopos medius & $\mathrm{N}$ & 27 & Muscicapa striata & $\mathrm{P}$ & 483 \\
\hline Dendrocopos leucotos & $\mathrm{N}$ & 6 & Ficedula hypoleuca & $\mathrm{N}$ & 251 \\
\hline Dendrocopos minor & $\mathrm{P}$ & 142 & Aegithalos caudatus & $\mathrm{P}$ & 613 \\
\hline Lullula arborea & SW & 643 & Parus palustris & $\mathrm{N}$ & 103 \\
\hline Anthus trivialis & $\mathrm{N}$ & 258 & Parus cristatus & $\mathrm{P}$ & 512 \\
\hline Erithacus rubecula & $\mathrm{P}$ & 566 & Parus ater & $\mathrm{P}$ & 529 \\
\hline Luscinia megarhynchos & SW & 728 & Parus caeruleus & $\mathrm{P}$ & 733 \\
\hline Phoenicurus phoenicurus & $\mathrm{P}$ & 245 & Parus major & $\mathrm{P}$ & 792 \\
\hline Turdus merula & $\mathrm{P}$ & 795 & Sitta europaea & $\mathrm{P}$ & 379 \\
\hline Turdus philomelos & $\mathrm{N}$ & 324 & Certhia familiaris & $\mathrm{N}$ & 52 \\
\hline Hippolais pallida & M & 113 & Certhia brachydactyla & SW & 689 \\
\hline Hippolais polyglotta & SW & 695 & Garrulus glandarius & $\mathrm{P}$ & 623 \\
\hline Sylvia undata & M & 655 & Fringilla coelebs & $\mathrm{P}$ & 738 \\
\hline Sylvia cantillans & M & 502 & Serinus serinus & $\mathrm{P}$ & 803 \\
\hline Sylvia melanocephala & M & 453 & Serinus citrinella & SW & 120 \\
\hline Sylvia hortensis & M & 369 & Carduelis chloris & $\mathrm{P}$ & 756 \\
\hline Sylvia communis & $\mathrm{P}$ & 512 & Carduelis spinus & $\mathrm{N}$ & 64 \\
\hline Sylvia borin & $\mathrm{N}$ & 406 & Loxia curvirostra & $\mathrm{P}$ & 299 \\
\hline Sylvia atricapilla & $\mathrm{P}$ & 627 & Pyrrhula pyrrhula & $\mathrm{N}$ & 212 \\
\hline Phylloscopus bonelli & SW & 556 & Coccothraustes coccothraustes & $\mathrm{P}$ & 157 \\
\hline
\end{tabular}

